\title{
Über ein Cyanid und eine Carbonsäure des Isochinolins
}

\author{
von \\ stud. phil. Berthold Jeiteles.
}

Aus dem chemischen Laboratorium der k. k. deutschen Universität in Prag.

(Vorgelegt in der Sitzung am 13. December 1894.)

Da bis nun, abgesehen von der von Goldschmiedt bei der Oxydation des Papaverins erhaltenen $B 2.3$. Dimethoxypy- $\alpha$-Isochinolincarbonsäure, ${ }^{1}$ noch keine Carbonsäure des Isochinolins bekannt ist, versuchte ich auf Anregung des Herrn Prof. Dr. Goldschmiedt, in dessen Laboratorium ein Cyanid der Base darzustellen und dasselbe $z u$ verseifen. Bedall und Fischer, ${ }^{2}$ La Coste, ${ }^{3} \mathrm{O}$. Fischer und Körner ${ }^{4}$ haben durch Destillation von o-chinolinsulfosaurem Kalium mit gelbem Blutlaugensalz, beziehungsweise Cyankalium o-Cyanchinolin, dem eine Spur der ana-Verbindung beigemengt war, erhalten. Durch deren Verseifung sind die genannten Autoren zu den entsprechenden Chinolinmonocarbonsäuren gelangt.

Ich verfuhr in ganz analoger Weise und erhielt so ein Cyanid des Isochinolins und bei dessen Verseifung eine Isochinolinmonoçarbonsäure, von der ich auch einige Derivate darstellte.

1 Monatshefte für Chemie, IX, S. 778.

2 B. B. XIV, 2574.

3 B. B. XV, 196.

4 B. B. XIII, 765. 


\section{Darstellung des isochinolinsulfosauren Natrons.}

Bereits Hoogewerff und van Dorp, die Entdecker des Isochinolins, haben eine Sulfurirung der Base vorgenommen in der Weise, dass sie Isochinolin mit concentrirter Schwefelsäure durch mehrere Tage in zugeschmolzenen Röhren auf dem Wasserbade erhitzten. Sie erhielten zwei isomere Sulfosäuren, die durch die verschiedene Löslichkeit ihrer Barytsalze getrennt wurden. Seeleman ${ }^{1}$ hat dieses Verfahren modificirt, indem er im offenen Kolben Isochinolin mit concentrirter Schwefelsäure, die $60 \%$ Anhydrid enthielt, bei Temperaturen von $110-112^{\circ}$ erhitzte. Bei den ersten Sulfonirungen verfuhr ich in dieser Weise, später jedoch nahm ich statt der anhydridreichen Säure tohe, rauchende Schwefelsäure des Handels, ohne dass die Ausbeute, die übrigens von der theoretischen weit entfernt ist, erheblich schlechter wurde. Nur musste die Temperatur bis $200^{\circ}$ erhoben werden. Es stimmt dies mit Seelemann's Angaben völlig üborein. Der Kolbeninhalt wurde in kaltes Wasser gegossen und mit kohlensaurem Baryt neutralisirt. Die abfiltrirte Lösung, die gelblich gefärbt war, wurde bedeutend eingeengt. Es fielen zuerst lange, reinweisse Nadeln aus, die abgesaugt wurden. Das Filtrat wurde ganz allmälig eingeengt und aus demselben krystallisirten noch beträchtliche Mengen weisser Nadeln aus. Endlich, nachdem selbst nach tagelangem Stehen im Exsiccator keine Krystalle anschossen, wurde zur 'Trockene verdampft und so das Baryumsalz der zweiten Sulfosäure in warzenförmigen Gebilden erhalten. Dieses ist in heissem Wasser schwer löslich, fällt aber, einmal in Lösung, nur sehr schwer heraus. Die Nadeln hingegen lösen sich in lauem Wasser sehr leicht und krystallisiren beim Erkalten aus. Doch zeigt sich auch hier oft Krystallisationsverzug. Bei der Behandlung mit rauchender Schwefelsäure bei $200^{\circ}$ wurde vornehmlich jene Sulfosäure erhalten, deren Barytsalz in Nadeln krystallisirt. Von dem Isomeren erhielt ich nur geringe Quantitäten. 
Aus dem in Nadeln krystallisirenden Baryumsalze wurde durch Natriumcarbonat das Natriumsalz dargestellt.

Es wurde auf dem Wasserbade scharf getrocknet und mit wasserfreiem, gelben Blutlaugensalz ( 2 Theile auf 1 Theil sulfosaures Natrium) aus einem knieförmig gebogenen Rohre im Wasserstoffstrome destillirt. Dieses Verfahren empfiehlt sich besser als die Destillation aus einer Retorte, da die Temperatur viel leichter regulirt werden kann. Es konnte jedoch auch unter diesen Verhältnissen eine theilweise Zersetzung des Cyanids in Isochinolin und Cyanwasserstoffsäure nicht ganz vermieden werden. Die Ausbeute betrug gewöhnlich $1 / 5$ bis $1 / \%$ der theoretischen.

Auch beim Chinolin konnte die Ausbeute nicht über $1 / \%$ der theoretischen erhoben werden. Das Destillationsproduct bildet ein gelbes bis röthliches Öl, das nach kurzer Zeit krystallinisch erstarrt. Ausserdem findet man in der Vorlage wohlausgebildete reinweisse Nadeln. Auch Ammoniak und Blausäure bilden sich, was am Geruche ganz deutlich erkannt wird. Das Destillat wurde in verdünntem Alkohol gelöst. Die Lösung ist grünlich, stark fluorescirend. Nach Wasserzusatz wurde die Lösung ins Vacuum gebracht. Es krystallisirten weisse Nadeln mit einem Stich ins Gelbe, zuweilen auch ins Grüne aus, die bei $134^{\circ}$, nach mehrmaligem Unkrystallisiren bei $135^{\circ}$ schmolzen (I). Das Cyanid lässt sich auch ohne verseift zu werden, aus heissem Wasser umkrystallisiren, in welchem es ziemlich schwer löslich ist. Es erstarrt beim Erkalten in kleinen, gelblichen Nadeln, nachdem die Flüssigkeit milchig geworden. (Analyse II bezieht sich auf die aus Wasser umkrystallisirte Substanz). Ganz rein lässt sich das Cyanid nur durch Sublimation erhalten, die schon bei $100^{\circ}$ zum Theile und bei $120^{\circ}$ ziemlich rasch von statten geht. Es stellt dann ganz reinweisse Nadeln dar (III).

I. $0 \cdot 1841 \mathrm{~g}$ lieferten $0.5229 \mathrm{~g} \mathrm{CO}_{2}$ und $0.0685 \mathrm{~g} \mathrm{H}_{2} \mathrm{O}$.

II. $0.1737 \mathrm{~g}$ lieferten $0.4928 \mathrm{~g} \mathrm{CO}_{2}$ und $0.0592 \mathrm{~g} \mathrm{H}_{2} \mathrm{O}$.

III. $0 \cdot 1601 \mathrm{~g}$ gaben $26 \cdot 5 \mathrm{~cm}^{3}$ feuchten Stickstoff bei $22^{\circ} \mathrm{C}$. und $751 \mathrm{~mm}$ Barometerdruck. 
In 100 Theilen:

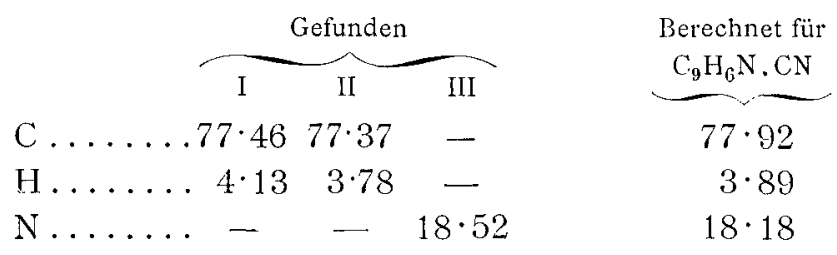

Das Cyanid ist in Säuren, selbst sehr verdünnten, äusserst leicht löslich, indem es mit denselben Salze bildet, in ähnlicher Weise wie Isochinolin selbst. Das salzsaure Salz bildet gelbliche Nadeln und ist in Wasser ausserordentlich leicht löslich. Mit Platinchlorid bildet es ein Doppelsalz, büschelförmige, gelbe Krystallnadeln, die, in heisser, concentrirter Salzsäure leicht löslich, beim Erkalten sich langsam abscheiden.

$0.1981 \mathrm{~g}$ der bei $100^{\circ}$ getrockneten Substanz lieferten, im Tiegel geglüht, $0 \cdot 0529 \mathrm{~g}$ metallisches Platin.

In 100 Theilen:

Pt. . . . $\underbrace{\text { Gefunden }}_{26.7} \quad \frac{\begin{array}{c}\text { Berechnet für } \\ \left(\mathrm{C}_{3} \mathrm{H}_{6} \mathrm{NCNHCl}_{2} \mathrm{PtCl}_{4}\right.\end{array}}{27.08}$

\section{Verseifung des Isochinolincyanids.}

Das Cyanid ist verhältnissmässig sehr beständig und nur sehr schwierig verseifbar. Selbst durch 6 Stunden fortgesetztes Kochen mit rauchender Salzsäure unter fortwährendem Ersatz der verdampften Säuremenge führte noch keine Verseifung herbei, ebenso zweistündiges Erhitzen mit concentrirter Salzsäure im zugeschmolzenen Rohre. Nach dem Neutralisiren mit Ammoniak fiel in beiden Fällen ein weisser Niederschlag aus, der den Schmelzpunkt des Cyanisochinolins zeigte. Erst dreistündiges Erhitzen mit rauchender Salzsäure bei 150-160 im zugeschmolzenen Rohre hatte den erwünschten Erfolg. Beim Öffnen der Röhre zeigte sich kein Druck. Der Röhreninhalt, vor dem Erwärmen eine röthlich gefärbte, klare Lösung, bestand nach dem Öffnen aus einer compacten Krystallmasse weisser 
Nadeln, dem salzsauren Salz der Isochinolinmonocarbonsäure. Nach mehrmaligem Umkrystallisiren aus heissem Wasser, worin die Nadeln äusserst leicht löslich sind, und scharfem Abpressen der röthlich gefärbten Mutterlauge, wurde die Substanz analysirt.

$0.3572 g$ der bei $100^{\circ}$ getrockneten Substanz gaben $0.2428 g$ $\mathrm{Ag} \mathrm{Cl}$.

In 100 Theilen:
Berechnet für
Gefunden
$\mathrm{C}_{9} \mathrm{H}_{6} \mathrm{NCO}_{2} \mathrm{H} . \mathrm{HCl}$
Cl .... 16:81
$16 \cdot 94$

$0.4171 \mathrm{~g}$ lufttrockener Substanz gaben bei anhaltendem Trocknen bei $100^{\circ}$ einen Verlust von $0.0599 \mathrm{~g} \mathrm{H}_{2} \mathrm{O}$, der zwei Molekülen Krystallwasser entspricht.

In 100 Theilen:

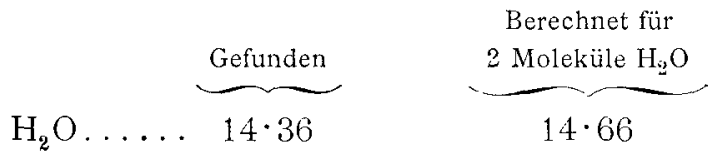

Die Substanz ist in Alkohol löslich und fällt auf Ätherzusatz aus.

Platindoppelsalz: Eine heisse, concentrirte Lösung in rauchender Salzsäure wurde mit Platinchlorid versetzt. Es fielen zum Theile schon in der Hitze, reichlicher beim Erkalten, gekrümmte, gelbe Nadeln aus, die beim Liegen an der Luft ihr glänzendes Aussehen bald verloren.

I. $0.2733 \mathrm{~g}$ der bei $100^{\circ}$ getrockneten Substanz gaben $0.3133 \mathrm{~g} \mathrm{CO}_{2}$ und $0.0573 \mathrm{~g} \mathrm{H}_{2} \mathrm{O}$.

II. $0.2527 \mathrm{~g}$ der bei $100^{\circ}$ getrockneten Substanz gaben, im Tiegel eingeäschert, $0.0653 g$ metallisches Platin.

In 100 Theilen:

\begin{tabular}{|c|c|c|}
\hline Gef & den & Berechnet für \\
\hline I & $\overline{\text { II }}$ & $\left(\mathrm{C}_{9} \mathrm{H}_{6} \mathrm{NCO}_{2} \mathrm{HHCl}\right)_{2} \mathrm{PtCl}_{4}$ \\
\hline$\ldots \ldots 31 \cdot 26$ & - & $31 \cdot 76$ \\
\hline . $2 \cdot 32$ & - & 2.11 \\
\hline - & $25 \cdot 86$ & $25 \cdot 72$ \\
\hline
\end{tabular}




\section{Darstellung der freien Säure.}

Das Chlorhydrat wurde zur Neutralisation der additionell gebundenen Salzsäure mit der berechneten Menge titrirten Ammoniaks versetzt. Ein Überschuss des letzteren musstevermieden werden, damit die Bildung eines Ammonsalzes der Säure verhindert werde. Es entstand sofort ein weisser Niederschlag, der in heissem Wasser sehr schwer löslich war, aber beim Erkalten der Lösung sich schwierig und nur theilweise in schwach gelbgefärbten Nadeln abschied. Ihr Schmelzpunkt liegt bei $272^{\circ}$, nachdem bei $247-250^{\circ}$ Schwarzfärbung auftritt. Die vom Niederschlage abfiltrirte Lösung wurde zur Trockene eingedampft, mit heissem Alkohol aufgenommen, der das gebildete Ammonchlorid ungelöst zurückliess. Die Säure ist in heissem Alkohol ziemlich schwer löslich und fällt beim Erkalten der Lösung in gut ausgebildeten Nadeln aus. Die Substanz gab beim Trocknen bei $100^{\circ}$ kein Wasser ab.

$0.2357 \mathrm{~g}$ lieferten $0.0850 \mathrm{~g}_{\mathrm{z}} \mathrm{O}$ und $0.5952 \mathrm{~g} \mathrm{CO}_{2}$.

In 100 Theilen:

\begin{tabular}{|c|c|c|}
\hline & Gefunden & $\begin{array}{l}\text { Bcrechnet für } \\
\mathrm{C}_{3} \mathrm{H}_{6} \times \mathrm{CO}_{2} \mathrm{H}\end{array}$ \\
\hline & $68 \cdot 87$ & $69 \cdot 36$ \\
\hline$[\ldots \ldots$ & $4 \cdot 00$ & 4.04 \\
\hline
\end{tabular}

Als Derivat des Isochinolins gibt die Substanz mit Säuren, als Carbonsäure mit Basen, Salze. Das salzs a ure und das salpetersaure Salz zeichnen sich durch prachtvolle Fluorescenz ihrer wässerigen Lösungen aus. Die wässerige Lösung des salzsauren Salzes ist röthlich gefärbt und fluorescirt bläulich, die des salpetersauren Salzes gelblich und fluorescirt grün.

Salpetersaures Salz. Durch Kochen der Säure mit concentrirter Salpetersäure und Eingiessen in kaltes Wasser wurde ein voluminöser, gelblich gefärbter Krystallbrei erhalten. Aus heissem Wasser, worin derselbe äusserst leicht löslich ist, schossen beim Erkalten grosse, büschelförmig aggregirte Nadeln an, die bei $218-220^{\circ}$ unter stürmischer Gasentwickelung schmolzen. Bei weiterem Erwärmen wurde der Inhalt der 
Capillare wieder fest und verflüssigte sich endlich bei $262^{\circ}$. Dass sich thatsächlich bloss ein salpetersaures Salz und keine Nitroverbindung gebildet, zeigte eine qualitative Prüfung auf Salpetersäure der bei $100^{\circ}$ getrockneten Substanz - mit Eisensulfat und concentrirter Schwefelsäure - in wässeriger Lösung, sowie eine Titration der Substanz mit $1 / 10$ normaler Natronlauge. $0 \cdot 2169 \mathrm{~g}$ Substanz verbrauchten zur Neutralisation $18 \cdot 9 \mathrm{~cm}^{3}$ $1 / 10$ normaler Natronlauge.

Verbrauch an $\mathrm{NaOH}$ :

$\underbrace{\text { Gefunden }}_{0.0756 g} \underbrace{\begin{array}{c}\text { Berechnet für } \\ \text { 2 Moleküle Säure }\end{array}}_{0.0735 g}$

$0.2383 \mathrm{~g}$ der bei $100^{\circ}$ getrockneten Substanz gaben $25 \mathrm{~cm}^{3}$ feuchten Stickstoff bei $21^{\circ} \mathrm{C}$. und $756.5 \mathrm{~mm}$ Barometerdruck.

In 100 Theilen:

$$
\text { N...... } \underbrace{\text { Gefunden }}_{11.89} \frac{\begin{array}{c}
\text { Berechnet für } \\
\mathrm{C}_{9} \mathrm{H}_{6} \mathrm{NCO}_{2} \mathrm{HHNO}_{3}
\end{array}}{11 \cdot 86}
$$

I. $0 \cdot 1715 g$ verloren bei $100^{\circ} 0 \cdot 0124 g \mathrm{H}_{2} \mathrm{O}$.

II. $0.2569 g$ verloren bei $100^{\circ} 0.0186 \mathrm{~g} \mathrm{H}_{2} \mathrm{O}$.

In 100 Theilen:

$$
\overbrace{\text { I. }}^{\text {Gefunden }} \underbrace{}_{\text {II. }} \mathrm{H}_{2} \ldots \ldots \cdot 7 \cdot 23 \quad \underbrace{1 \text { Molekü1 } \mathrm{H}_{2} \mathrm{O}}_{7 \cdot 24}
$$

Pikrinsäureverbindung der Isochinolincarbonsäure. Eine heisse, alkoholische Lösung der Säure wurde mit der molecularen Menge Pikrinsäure, ebenfalls in heisser, alkoholischer Lösung versetzt. Beim Erkalten fielen gelb gefärbte Krystalle aus, die aus heissem Alkohol unter Zusatz von etwas Pikrinsäure umkrystallisirt wurden. Ihr Schmelzpunkt lag bei $212-213^{\circ}$.

$0.1669 \mathrm{~g}$ Substanz gaben $20 \mathrm{~cm}^{3}$ feuchten Stickstoff bei $22^{\circ} \mathrm{C}$. und $755.5 \mathrm{~mm}$ Barometerdruck. 
In 100 Theilen:

$$
\mathrm{N} \ldots \ldots \underbrace{\text { Gefunden }}_{13 \cdot 49} \quad \underbrace{\mathrm{C}_{9} \mathrm{H}_{6} \mathrm{NCO}_{2} \mathrm{HC}_{6} \mathrm{H}_{2}\left(\mathrm{NO}_{2}\right)_{3} \mathrm{OH}}_{13 \cdot 93}
$$

Kupfersalz: Eine heisse, wässerige Lösung der Säure wurde mit kupferacetat unter Zusatz weniger Tropfen Essigsäure versetzt. Es fiel sofort ein blauer, krystallinischer Niederschlag aus, der in heissem Wasser unlöslich war. Er wurde abfiltrirt, sorgfältig gewaschen, bei $100^{\circ}$ getrocknet und der Analyse unterworfen.

$0.1215 g$ gaben $0.0415 g \mathrm{H}_{2} \mathrm{O}$ und $0.2599 g \mathrm{CO}_{2}$.

In 100 Theilen:

\begin{tabular}{|c|c|c|}
\hline & Gefunden & $\begin{array}{c}\text { Eerechnet fur } \\
\left(\mathrm{C}_{9} \mathrm{H}_{6} \mathrm{NCO}_{2}\right)_{3} \mathrm{Cu}\end{array}$ \\
\hline C. & $58 \cdot 35$ & $58 \cdot 93$ \\
\hline $\mathrm{H}$ & $3 \cdot 79$ & $2 \cdot 94$ \\
\hline
\end{tabular}

Bromadditionsproduct. Eine kalte, gesättigte Lösung der Säure wurde mit Bromwasser versetzt. Es fiel sofort ein amorpher, gelblichrother Niederschlag aus, der mit kaltem Wasser gewaschen wurde. Die Substanz wurde über Kalk und Schwefelsäure gestellt und gab das lose gebundene Brom successive $a b$, was sich an dern ganz deutlichen Bromgeruch zeigte, den die Substanz selbst nach mehrwöchentlichem Stehen im Exsiccator noch hatte. Von einer Analyse musste bei dieser Unbeständigkeit des Körpers abgesehen werden. Beim Kochen mit Wasser gab der Körper sein Brom vollständig ab und es konnte nachgewiesen werden, dass Isochinolincarbonsäure wieder entstand.

\section{Oxydation der Isochinolincarbonsäure mit Kaliumper- manganat.}

Isochinolin und seine Derivate liefern, wie Hoogewerff und van Dorp, ${ }^{1}$ später Goldschmiedt ${ }^{2}$ gezeigt, bei Oxydation mit Chamaeleonlösung bei Gegenwart freier Säure Phtalsäure, respective ihre Derivate, und Cinchomeronsäure; in neutraler Lösung, wie Goldschmiedt beobachtet, Phtalimid

1 Recueil des trav. chim. des Pays Bas. IV, p. 285.

2 Monatshefte für Chemie, IX, 67\%. 
und Cinchomeronsäure. Dieses Verhalten bietet bekanntlich ein Mitfel, aus den Oxydationsproducten auf die Constitution der substituirten Isochinoline einen Schluss zu ziehen. Um nun die Stelle, wo die Carboxylgruppe in den Isochinolinring eingetreten war, sicherzustellen, wurden Oxydationen sowohl in saurer, als auch neutraler Lösung vorgenommen und auch die Temperatur bei verschiedenen Operationen verschieden gewählt. Die Ausbeute war in allen Fällen eine äusserst schlechte, so dass $16 \mathrm{~g}$ oxydirt werden mussten, um nur die zur Analyse nothwendige Menge des Oxydationsproductes zu erhalten.

Ich erhielt, wie aus Späterem ersichtlich, eine Benzoltricarbonsäure, die in ihren Eigenschaften mit der von v. Baeyer ${ }^{\prime}$ entdeckten Hemimellithsäure eine so grosse Übereinstimmung zeigt, dass ich nicht Anstand nehme, sie für identisch mit derselben zu erklären. Die Oxydation wurde unter folgenden Bedingungen vorgenommen. Es wurden $4 g$ der Isochinolincarbonsäure in $1 l$ Wasser suspendirt und durch kohlensaures Natrium in Lösung gehalten. Unter Erwärmen auf $100^{\circ}$ wurde allmälig 4-procentige Kaliumpermanganatlösung eingetragen und die Lösung durch Einleiten von Kohlensäure, sowie zeitweises Eingiessen verdünnter Salzsäure möglichst neutral gehalten. Nach Verbrauch von $20-25 \mathrm{~g}$ Kaliumpermanganat schien die Oxydation beendigt, da auf weiteren Zusatz von Chamaeleon keine Entfärbung eintrat. Die geringe Menge des überschüssigen Chamaeleons wurde durch schweflige Säure entfärbt. Es wurde nun vom Braunstein abfiltrirt und letzterer mehrmals mit Wasser ausgekocht. Die Waschflüssigkeiten wurden vereinigt und auf dem Wasserbade eingeengt. Hierauf wurde mit verdünnter Schwefelsäure angesäuert und so lange mit Äther, dem etwas Alkohol beigemischt war, ausgeschüttelt, als derselbe noch etwas aufnahm. Nach dem Abdunsten desselben hinterblieben weisse Krystalle, die auffallenderweise einen nicht unbeträchtlichen Aschengehalt zeigten. Sie wurden mit etwas verdünnter Schwefelsäure gekocht und umkrystallisirt. Die erste Fraction bildete bräunlich gefärbte Blättchen, die ebenfalls noch Aschenrückstand mit alkalischer Reaction aufwiesen. Die Sub-

1 Annalen, Supplement 7, S. 31. 
stanz sinterte bei $259^{\circ}$ und schmolz bei $275^{\circ}$ unter Gasentwickelung. Es wurde ein neutrales Silbersalz dargestellt, indem die Substanz mit Ammoniak eingedunstet wurde, wobei sich strahlige Krystallnadeln zeigten, und dieses Ammonsalz heiss mit salpetersaurem Silber gefällt. Das Silbersalz stellt mikroskopische, feine Nadeln dar, die in Wasser fast unlöslich sind. Das direct durch Fällung gewonnene und gewaschene Salz wurde analysirt.

$0 \cdot 1657 \mathrm{~g}$ der bei $100^{\circ}$ getrockneten Substanz lieferten $0 \cdot 1180 \mathrm{~g}$ $\mathrm{CO}_{2}, 0 \cdot 0199 \mathrm{~g} \mathrm{H}_{2} \mathrm{O}$ und $0.0992 \mathrm{~g} \mathrm{Ag}$.

In 100 Theilen:
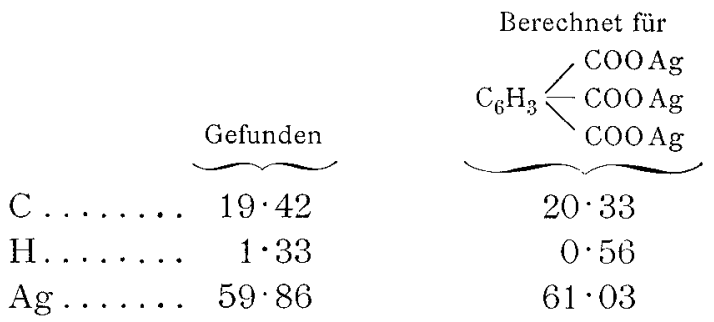

Nach dieser Analyse stellt sich die Substanz als neutrales Silbersalz einer Benzoltricarbonsäure dar, wenn auch nicht in ganz reinem Zustande.

Der Rest der Substanz wurde mit wenigen Tropfen verdünnter Schwefelsäure auf dem Wasserbade bis zur Syrupdicke eingeengt und in Alkohol gegossen. Hiebei fiel schwefelsaures Kali aus. Die Substanz, die in Lösung ging, wurde nach dem Abdunsten des Alkohols aus Wasser umkrystallisirt. Sie fällt sehr langsam aus und bildet reinweisse Blättchen, die bei $175^{\circ}$ zu sintern beginnen und bei $189^{\circ}$ flüssig werden. Im Röhrchen wurde ein nadelförmiges Sublimationsproduct (Phtalsäure) beobachtet und auch Benzoësäuregeruch trat beim Erhitzen auf. Nach längerem Erhalten auf dieser Temperatur wurde als Schmelzpunkt $120^{\circ}$ beobachtet.

Die Substanz gab mit basischem Bleiacetat einen flockigen, in Essigsäure unlöslichen Niederschlag. Das Ammonsalz krystallisirte strahlig. Das Silbersalz verpuffte beim Erhitzen. Mit Barythydrat entstand ein voluminöser Niederschlag, der, aus heissem Wasser umkrystallisirt, Prismen darstellte. Um die 
Identität dieser Substanz mit Hemimellithsäure zu verdeutlichen, stelle ich das Verhalten derselben mit den von v. Baeyer gemachten Angaben über Hemimellithsäure in Parallele.

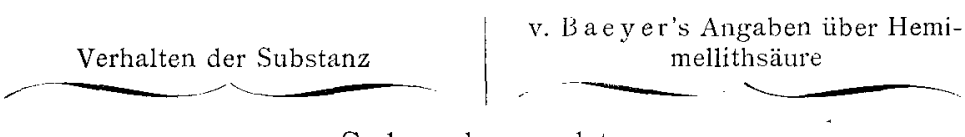

Schmelzpunkt:

Bei $175^{\circ}$ Sinterung, $189^{\circ}$ Verflüssigung. Nach längerem Erhalten auf dieser Temperatur Schmelzpunkt $120^{\circ}$; nadelförmiges Sublimationsproduct, Benzoësäuregeruch.
$»$ Sie fängt bei $185^{\circ}$ etwa an zu schmelzen, ein Theil bleibt aber noch weit über dieser 'Temperatur fest. Hält man sie längere Zeit geschmolzen, so erstarrt sie zu einer krystallinischen Masse, die schon bei $125^{\circ}$ schmilzt. Während des Schmelzens sublimiren lange Nadeln, welche die grösste Ähnlichkeit mit Phtalsäureanhydrid zeigen. Zu gleicher Zeit nimmt man den Geruch von Benzoësäure wahr

Bleiacetat:

In Essigsäure unlöslicher flockiger Niederschlag.
"Flockiger Niederschlag, der in überschüssiger Essigsäure schwer löslich ist «.

\section{Ammonsalz:}

Strahlig krystallinisch, in Wasser sehr leicht löslich.
"Ist in Wasser leicht löslich, krystallisirt beim Verdunsten auf dem Uhrglase strahlig «.

\section{Silbersalz:}

Verpufft beim Erhitzen.

»Beim schnellen Erhitzen verpufft das Silbersalz . 


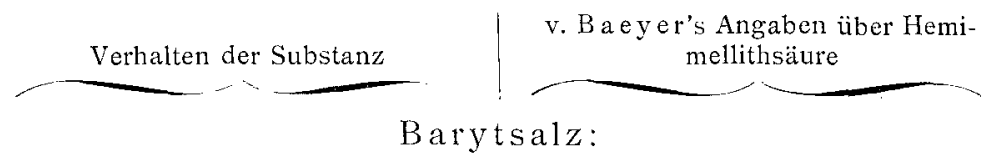

Aus der wässerigen Lö»Barytwasser fällt eine sung der Säure fällt Barytwasser einen weissen Niederschlag, der nach dem Umkrystallisiren Nadeln bildet.

Es erscheint somit Hemimellithsäure als Oxydationsproduct dieser Isochinolincarbons äure. Hieraus folgt, dass die Carboxylgruppe in den Benzolkern, und zwar entweder in $o$ - oder ana-Stellung zum Stickstoffatom eingetreten ist. Die Oxydation wäre nach folgendem Schema verlaufen.

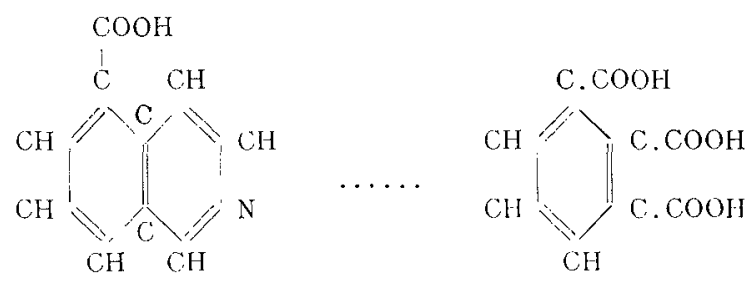

oder<smiles>O=C(O)c1cccc2ccccc12</smiles><smiles>O=C(O)c1ccc(C(=O)O)c(C(=O)O)c1</smiles>

Claus und Raps ${ }^{1}$ haben für die Sulfosäure, deren Barytsalz zuerst in langen Nadeln ausfällt, welche also die von mir verwendete war, die $\beta$-Stellung der Sulfogruppe für wahrscheinlich gehalten. Sie stützten sich hiebei auf theoretische Erwägungen unter Zugrundelegung der Claus'schen Isochinolinformel und auf das Verhalten der aus den Sulfosäuren gewonnenen Oxyisochinoline. Später hat aber Gutzeit ${ }^{2}$ im

1 J. f. pr. Ch. 45 . S. 241.

2 J, f. pr. Ch. 47 . S. 437. 
Claus'schen Laboratorium aus dem Amidoisochinolin, für welches durch die Untersuchungen von Claus und Hoffmann, ${ }^{1}$ sowie durch die im hiesigen Laboratorium von Fortne ${ }^{2}$ ausgeführten Versuche übereinstimmend die $o$ - oder ana-Stellung sichergestellt ist, ein Oxyisochinolin dargestellt, welches dem früher aus der Sulfosäure bereiteten »zum Verwechseln ähnlich ist «. Daraus würde sich ergeben, dass Claus jetzt für diese Sulfosäure die Stellung o- oder ana- für nicht unwahrscheinlich hält, was auch mit dem, bei der Oxydation der aus dieser Sulfosäure dargestellten Isochinolincarbonsäure, gewonnenen Resultate in Übereinstimmung stehen würde.

Bei anderen Oxydationen, die bei $60-80^{\circ}$, unter strenger Wahrung der Neutralität, vorgenommen wurden, wurde im Ätherauszuge in geringer Menge eine Substanz erhalten, die bei etwa $100^{\circ}$ sinterte, bei $110-130^{\circ}$ unter Gasentwickelung sich verflüssigte, bei $180^{\circ}$ wieder fest wurde und endlich gegen $225^{\circ}$ schmolz. Beim Kochen dieser Substanz mit Kalilauge wurde qualitativ Bildung von A m moniak nachgewiesen, was für die Bildung eines Imids bei der Oxydation der Isochinolincarbonsäure spricht. Der Schmelzpunkt von $225^{\circ}$, der dem Phtalimid entspricht, sowie das Verhalten der Substanz bei höherer Temperatur deutet wohl darauf hin, dass eine, wenn auch etwas verunreinigte, Phtalimidcarbonsäure vorliege, die, wie es ja sehr wahrscheinlich ist, Kohlensäure abspaltet, um endlich in Phtalimid überzugehen.

Die mit Äther erschöpfte, noch saure Oxydationsflüssigkeit wurde mit kohlensaurem Natrium genau neutralisirt, eingedampft und in das doppelte Volum Alkohol eingegossen. Es wurde von den ausgeschiedenen Mineralsalzen abfiltrirt, der Alkohol verdampft und der wässerige Rückstand mit überschüssigem Kupferacetat versetzt. Es entstand ein hellblauer, krystallinischer Niederschlag, der sich beim Kochen noch vermehrte, im Ganzen aber doch nicht beträchtlich war. Das Kupfersalz wurde in Wasser suspendirt, durch Schwefelwasserstoff gefällt und die vom Schwefelkupfer filtrirte Lösung, die röth-

1 J. f. pr. Ch., S. 265.

2 Monatshefte für Chemie, XIV. 146. 
lich gefärbt war, eingedunstet. Es fiel eine geringe Menge brauner Krystalle aus, die auch nach mehrmaligem Umkrystallisiren nicht rein wurden. Dementsprechend ist auch der Schmelzpunkt nicht scharf, und es ist nicht fraglich, dass ein Gemenge vorliegt. Auf dessen Trennung musste wegen der geringen Menge verzichtet werden, doch machen es einige Versuche im höchsten Grade wahrscheinlich, dass der Hauptbestandtheil dieser Substanz, die hier zu erwartende Cinchomeronsäure ist. Dafür spricht, dass die Substanz in Salzsäure löslich ist und dass aus dieser Lösung prismatische, gelbe Krystalle erhalten werden können; ferner, dass eine Probe der Säure, mit Kalk destillirt, ein nach Pyridin riechendes Öl gibt, das, mit Salzsäure und Platinchlorid versetzt, ein Doppelsalz liefert, welches unter dem Mikroskope ganz so aussieht, wie das aus reinem Pyridin unter gleichen Verhältnissen dargestellte Chlorplatinat. 